\title{
Salud Mental Positiva. \\ Cuidar la Salud Mental Positiva, en el contexto \\ de la pandemia COVID-19 y hacía un futuro denominado "nueva normalidad"
}

María Teresa Lluch Canut *

\section{Resumen}

El objetivo general de este artículo es el de compartir conocimientos entorno a la Salud Mental Positiva y reflexiones de su importancia en el contexto actual de pandemia COVID-19 y hacía un futuro inmediato denominado "nueva normalidad". Tras una introducción, se desarrolla una definición básica de Salud Mental Positiva (SMP) que permite identificar la necesidad de aceptar como mentalmente saludable pensamientos, sentimientos y comportamientos positivos y negativos. Pero se detallan de forma relevante los indicadores límite de alerta de Salud Mental Positiva, a los que hay que prestar especial atención y pedir ayuda si es necesario: duración, intensidad, frecuencia, diferencia en el patrón habitual de respuesta, y consecuencias. Posteriormente, se describe el Modelo Multifactorial de Salud Mental Positiva-MMSMP de Lluch Canut (1999), que concibe la SMP como un constructo configurado por 6 factores: Satisfacción Personal (F1), Actitud Prosocial (F2), Autocontrol (F3), Autonomía (F4), Resolución de Problemas y Autoactualización (F5) y Habilidades de Relación Interpersonal (F6). El MMSMP se evalúa mediante un cuestionario creado por la misma autora (Cuestionario de Salud Mental Positiva-CSMP) constituido por 39 ítems, distribuidos de forma desigual entre los 6 factores del modelo MMSMP. Se citan diversos estudios vinculados al tema, tanto a nivel de investigaciones psicométricas, como estudios correlacionales y programas de intervención para la promoción y la prevención de la Salud Mental Positiva, en diversas poblaciones (estudiantes universitarios, personas con problemas crónicos de salud física, cuidadoras de personas con problemas crónicos de salud física y/o mental, entre otros). También se describen estrategias para cuidar la Salud Mental Positiva, en base a Decálogos, adaptados a la situación específica de pandemia COVID-19. Y por último se exponen unas reflexiones a modo de conclusiones, en dos puntos principales: la Salud Mental Positiva en el contexto de desarrollo existencial, y la Salud Mental Positiva y el Sufrimiento Emocional.

Palabras clave: $\quad$ Salud Mental Positiva - Modelo Multifactorial de Salud Mental Positiva - Sufrimiento Emocional - Pandemia COVID-19 Indicadores Límite de Alerta de Salud Mental Positiva 


\title{
Positive Mental Health. Caring for Positive Mental Health, in the context of the COVID-19 pandemic and towards a future called "new normal"
}

\author{
María Teresa Lluch Canut *
}

\begin{abstract}
The general objective of this article is to share knowledge about Positive Mental Health and reflections on its importance in the current context of the COVID-19 pandemic and towards an immediate future called "new normal". After an introduction, a basic definition of Positive Mental Health (SMP) is developed that allows identifying the need to accept positive and negative thoughts, feelings and behaviors as mentally healthy. But the warning threshold indicators of Positive Mental Health are detailed in a relevant way, to which we must pay special attention and ask for help if necessary: duration, intensity, frequency, difference in the usual pattern of response, and consequences. Subsequently, the Multifactorial Model of Positive Mental HealthMMSMP by Lluch Canut (1999) is described, which conceives the SMP as a construct configured by 6 factors: Personal Satisfaction (F1), Prosocial Attitude (F2), Self-control (F3), Autonomy (F4), Problem Solving and Self-actualization (F5) and Interpersonal Relationship Skills (F6). The MMSMP is evaluated using a questionnaire created by the same author (Positive Mental Health Questionnaire-CSMP) consisting of 39 items, unevenly distributed among the 6 factors of the MMSMP model. Various studies related to the subject are cited, both at the level of psychometric research, as well as correlational studies and intervention programs for the promotion and prevention of Positive Mental Health, in various populations (university students, people with chronic physical health problems, caregivers of people with chronic physical and / or mental health problems, among others). Strategies to care for Positive Mental Health are also described, based on Decalogues, adapted to the specific situation of the COVID-19 pandemic. And finally, some reflections are presented as conclusions, on two main points: Positive Mental Health in the context of existential development, and Positive Mental Health and Emotional Suffering.
\end{abstract}

Keywords: Positive Mental Health - Multifactorial Model of Positive Mental Health - Emotional distress - COVID-19 pandemic - Limit Positive Mental Health Alert Indicators 


\section{Introducción}

En el momento que recibí la invitación para participar en el número monográfico de la revista Hermenéutica "paradigma de la salud y cuidado" no conocíamos el coronavirus SARS-CoV-2 ni sus efectos a nivel global, ya sea desde la perspectiva planetaria, ya sea desde el microespacio personal de cada ser humano. Con la situación de pandemia COVID-19 estamos delante de un reto mental de gran importancia y envergadura. Si dejamos ir nuestra mente, nos encontramos con la necesidad de pensar y reflexionar acerca de en qué espacio podríamos colocar los pensamientos, los sentimientos y las emociones que las personas estamos viviendo y experimentando en este momento actual de vida, en que la humanidad está inmersa en la continuidad de la vida y, a la vez, en asumir lo que algunos autores están denominando la nueva normalidad de vida. Esa nueva normalidad que implica el uso de mascarillas, el mantenimiento de la distancia física en las interacciones de las personas, y un conjunto de amenazas o incertidumbres que generan muchas emociones y pensamientos de temor, preocupación o miedo. Sin obviar a todas las personas que se ven afectadas de un modo "dramático" por la pandemia COVID-19, ya sea por pérdida de la propia salud o la de sus seres queridos, ya sea por fallecimiento de personas allegadas, ya sea por pérdida de trabajo, 0 por complicaciones económicas, sociales, y una larga lista de aspectos y determinantes que hacen que cada persona, en su microcosmos este sometida a una prueba de "alto voltaje" en su salud mental positiva.

Hace años que inicie los estudios en el concepto de Salud Mental Positiva, buscando dos aspectos complementarios. El primer aspecto hacía referencia a la necesidad de explorar a nivel conceptual algo que en mi experiencia clínica constataba diariamente y era, y es, que, en cualquier situación en que se encuentre el ser humano, por compleja que sea, el modo en que cada persona afronta esa situación condiciona la vivencia personal de la misma y, por ende, el efecto que eso tiene en el propio entorno personal, familiar y social. Cuando conocí el concepto de Salud Mental Positiva, a través del documento elaborado por Marie Jahoda en 1958, en el marco de la preparación de la reforma comunitaria de la salud mental en Estados Unidos de Norteamérica, me pareció que era un concepto que podía conducirme a esa parte de la mente que da fuerza y energía para afrontar las adversidades y salir de ellas, sino resiliente, si de un modo aceptablemente saludable a nivel mental.

El segundo aspecto que buscaba en el estudio de la Salud Mental Positiva está vinculado con la definición originaria de salud mental, cuando se indica que "la salud mental es algo más que ausencia de enfermedad", ese espacio dedicado a la promoción, al refuerzo y al mantenimiento de la salud mental, a partir de la ausencia de trastorno (Lluch-Canut, 2015). Aunque la promoción es el espacio de desarrollo por excelencia para el mantenimiento y el refuerzo del bienestar mental, el ámbito de la Salud Mental Positiva traspasa la promoción y está inmerso en todos los ámbitos clínicos de la salud mental, tanto en prevención como en tratamiento, rehabilitación o recovery. Y sea en el ámbito 
que sea, el concepto Salud Mental Positiva explica de lleno ese "algo más que ausencia de enfermedad" pero también ese "espacio que se conserva de salud mental dentro de la enfermedad mental"

\section{Definición básica de Salud Mental Positiva e Indicadores límite de alerta}

Partiendo de una definición de la Salud Mental Positiva como "un conjunto de sentimientos, pensamientos y comportamientos positivos y negativos; a pesar de que hay que potenciar estados de bienestar y felicidad también hay que aceptar estados de malestar, entendiendo que en muchas ocasiones lo verdaderamente sano mentalmente, la verdadera salud mental positiva, es estar triste, decepcionado o preocupado" (LluchCanut, 1999, 2003, 2008, 2015).

Eso es así y es importantísimo tenerlo presente. El ser humano viene programado para experimentar todo tipo de emociones y para tener múltiples pensamientos. $Y$ todos ellos son válidos y sanos. Frente a situaciones como la pandemia del COVID-19 es muy sano mentalmente que las personas experimenten miedo, temor, preocupación y muchos más sentimientos, pensamientos y emociones negativas y de preocupación. No sería normal que se estuviera pasando por la situación de gravedad que existe sin experimentar, en mayor o menor medida, algunos o varios de los sentimientos negativos que podríamos listar y que, en definitiva, van vinculados a las emociones primarias y secundarias del ser humano, en su estructura psicológica sana.

Todo es válido, pero hay unos límites entre la normalidad y lo que no es normal. Y esos son los límites que hay que intentar no sobrepasar y estar atentos. Para ello puede ser de utilidad considerar lo que personalmente denomino: "Indicadores límite de alerta de Salud Mental Positiva" (Lluch-Canut 2008).

Reflexionando acerca de la denominación, el término "alerta" puede tener unas connotaciones negativas, lo cual podríamos decir que puede ir en contra de la filosofía global del término Salud Mental Positiva, que parece que siempre conduce a remansos de paz y bienestar. Pero creo que es acertado utilizar este término de "alerta" porque el concepto Salud Mental Positiva no es del todo benigno. Su expresión puede conducir a engaño, porque si lo que se pretende es que las personas siempre estén felices, contentas, piensen en positivo, sientan bienestar, ganas de hacer muchas cosas bonitas, etc., se corre el riesgo de conducir a las personas a un estado de patología o malestar. ¿Por qué? Por qué hay que aceptar que las personas a lo largo del día y a lo largo de nuestra vida pasamos momentos, días y periodos de sufrimiento, de preocupación y de malestar. Muchos de estos momentos o periodos van ligados a cosas y eventos que suceden en nuestra vida, interior y/o exterior. Los eventos vitales, aquellos por los que pasa el ser humano a lo largo de su vida, están identificados como fuentes especiales de funcionamiento extra de las capacidades del ser humano. Algunas personas pasan por las situaciones vitales con poca dificultad, otras salen reforzadas, pero en general, cualquier 
evento vital (pérdidas y muertes de seres queridos, cambios de ciclo evolutivo vinculados a la edad, alteraciones de la salud, etc.) pone en funcionamiento la demanda de la mente para tirar adelante: la energía mental.

\section{¿Cuáles son los “indicadores límite de alerta de Salud Mental Positiva?}

De lo expuesto anteriormente, se deduce y se acepta que todo comportamiento, pensamiento y sentimiento positivo y negativo es sano mentalmente. Pero con límites, y esos límites son 6:

- La duración: cuando una reacción o un sentimiento, especialmente de carácter negativo y bloqueante, se prolonga mucho en el tiempo, puede hacer pensar en que quizás algo no va bien.

- La intensidad: cuando la intensidad de la respuesta lleva a la pérdida del control que la persona tiene sobre sus reacciones emocionales, puede indicar que algo no va bien.

- La frecuencia: aunque es importante vivir y sentir todo tipo de sentimientos y pensamientos, si estos son de carácter negativo y son muy frecuentes, habrá que prestarles especial atención porque puede indicar que algo no va bien.

- La incapacidad: cuando las respuestas emocionales y/o los pensamientos afectan al desarrollo de las necesidades básicas (dificultades para dormir, pérdida de apetito, desinterés por el entorno,...) e impiden la funcionalidad de la persona, puede indicar que algo no va bien.

- La diferencia en el patrón habitual de respuesta de la persona: cuando la reacción o la respuesta emocional que tiene la persona es muy diferente de la forma habitual que tiene esa misma persona en responder, puede indicar que algo no va bien; en cambio en otras ocasiones, respuestas emocionales o comportamientos que pueden sorprender a la mayoría de personas, ya se han dado en otras ocasiones en la persona y forman parte de su patrón "habitual" de respuesta; en ese caso pueden ayudar a interpretar que, aunque pueda parecer extraña la reacción, todo parece que puede ir bien.

- Las consecuencias: cuando la respuesta emocional o el comportamiento de la persona puede tener consecuencias negativas para sí mismo y/o para los demás, hay que valorar que quizás algo no va bien.

Estos "indicadores límite de alerta de Salud Mental Positiva" no son de carácter diagnóstico (no sirven para diagnosticar problemas o trastornos de salud mental) sino que actúan de señales de alerta, señales de aviso, para indicar que si eso ocurre hay que consultar a un/una profesional de la salud mental (ya sea una enfermera especialista en salud mental, un psicólogo/a o un psiquiatra). La puerta de entrada en la consulta no es determinante. También, en ocasiones, la ayuda no profesional de un familiar, un amigo o un colega puede ser suficiente. Lo importante es vigilar para valorar si la respuesta (física, mental o conductual) es indicativa de buena salud mental positiva o bien se está en 
situación de riesgo de padecer una alteración de la salud mental. Ante la duda hay que consultar.

La Salud Mental Positiva como constructo - Modelo Multifactorial de Salud Mental Positiva (Lluch-Canut, 1999)

El Modelo Multifactorial de Salud Mental Positiva (MMSMP) fue planteado por primera vez por Lluch-Canut en 1999. En el marco de este modelo, la Salud Mental Positiva (SMP) se concibe como un constructo. Implica que no tiene una definición directa y precisa de factores o dimensiones para su definición.

Según este planteamiento, la SMP se define a partir de 6 factores interrelacionados que configuran el MMSMP (Figura 1): Factor F1-Satisfacción Personal: hace referencia a la satisfacción con uno mismo (autoconcepto / autoestima), con la vida personal que uno tiene y con las perspectivas de futuro. Factor F2-Actitud Prosocial: incluye la sensibilidad de la persona hacía su entorno social, la actitud y el deseo de ayudar-apoyar a los demás y la aceptación de los demás y de los hechos sociales diferenciales. Factor F3-Autocontrol: contempla la capacidad de la persona para el afrontamiento del estrés y de situaciones conflictivas, el equilibrio emocional, y la tolerancia a la frustración, a la ansiedad y al estrés. Factor F4-Autonomía: incluye la capacidad de la persona para tomar sus propias decisiones aplicando criterios propios, autorregulando la propia conducta y manteniendo un buen nivel de seguridad personal. Factor F5-Resolución de Problemas y Autoactualización: hace referencia a la capacidad de la persona para tomar decisiones e ir resolviendo los problemas que la vida conlleva, así como la capacidad para adaptarse a los cambios, desarrollando una actitud flexible y de crecimiento personal continuo. Factor F6Habilidades de Relación Interpersonal: incluyen la capacidad de la persona para comunicarse y establecer relaciones interpersonales armoniosas con su entorno y la habilidad para comunicar sentimientos y dar y recibir afecto.

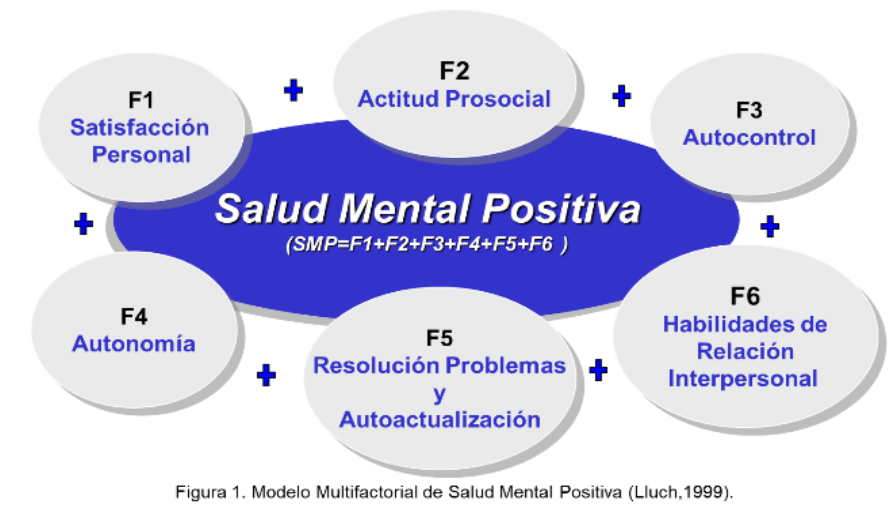

En la bibliografía contemporánea los factores que configuran este modelo aparecen con frecuencia como dimensiones de los instrumentos de evaluación de la salud mental (por ejemplo, satisfacción o autonomía) y como objetivos a potenciar o entrenar 
en los programas de prevención y promoción (por ejemplo, habilidades sociales, habilidades para resolver problemas o estrategias que favorecen el autocontrol). Además, existen trabajos que describen relaciones parciales entre dos o tres factores (por ejemplo: la satisfacción personal con la autonomía o con el autocontrol). Sin embargo, no se ha localizado ningún otro autor que integre los seis factores en un marco conceptual y les confiera la categoría de modelo.

El Modelo Multifactorial de Salud Mental Positiva se basa en los postulados que se han descrito en párrafos anteriores acerca de la Salud Mental Positiva y que pueden sintetizarse en cuatro postulados (Lluch-Canut, 1999, 2002, 2003, 2008, 2015; LluchCanut, Sequeira \& Roldan-Merino, 2017):

La salud mental positiva es un estado dinámico y fluctuante: unos días estamos más animados y nos sentimos mejor y otros días estamos más cansados y nos sentimos más desanimados.

La salud mental positiva engloba sentimientos, pensamientos y comportamientos positivos y negativos. En este sentido es igual de saludable reír y sentirse contento cuando están pasando cosas buenas que llorar y estar triste cuando se presentan problemas, pérdidas o decepciones.

La salud mental positiva tiene unos límites que hay que controlar. Son los "indicadores límite de alerta de Salud Mental Positiva", descritos anteriormente: duración, intensidad, frecuencia, incapacidad, diferencia en el patrón habitual de respuesta de la persona y consecuencias.

Los factores que configuran el Modelo Multifactorial de Salud Mental Positiva (MMSMP) están interrelacionados de forma que unos pueden compensar a los otros, y así, en función del momento o de la situación personal, predominan unos factores sobre los otros. Por supuesto, para cada situación y cada momento de la vida de la persona hay una combinación idónea de factores: en ocasiones quizás el nivel de satisfacción con la vida personal es bajo, pero se puede mantener elevada la capacidad de resolver problemas. En otras ocasiones quizás el nivel de autocontrol es menor pero la capacidad de establecer y mantener relaciones interpersonales es buena, equilibrando el menor autocontrol emocional. En el apartado de evaluación de la Salud Mental Positiva señalamos algunos resultados de estudios donde hay determinantes sociodemográficos y de salud que identifican el mayor peso de algunos factores en el índice global de Salud Mental Positiva. En esta situación de pandemia de COVID-19 será interesante valorar si existen cambios en el predominio de los factores y en los valores globales de Salud Mental Positiva.

\section{Evaluación de la Salud Mental Positiva}

Para evaluar el Modelo Multifactorial de Salud Mental Positiva, Lluch-Canut (1999) construyó un Cuestionario (Cuestionario de Salud Mental Positiva-CSMP) configurado por 39 ítems distribuidos entre los 6 factores del modelo MMSMP. 
Tal y como se ha indicado, los factores del modelo MMSMP interrelacionan y se influencian mutuamente generando la posibilidad de obtener un nivel global de Salud Mental Positiva (a partir de la suma obtenida en los seis factores) y niveles de SMP para cada factor del modelo. Esta característica permite conocer de forma más detallada los factores y decidir si se desea intervenir en alguno de los factores específicos, o bien en todos. EI CSMP ha sido validado en diversas poblaciones presentando buenas propiedades psicométricas (Albacar-Riobóo, 2014; Hurtado, 2017; Hurtado et al., 2017, 2018; LluchCanut et al., 2013; Mantas Jiménez, 2017; Mantas Jiménez et al., 2015; Miguel, 2014; Roldán et al., 2017; Sánchez, 2015; Sanromá Ortiz, 2016; Sequeira et al., 2019) y ha sido traducido y publicado en portugués (Sequeira et al., 2014) y en turco (Teke \& Baysan, 2018).

Si bien aún no se dispone de baremos normativos poblacionales de Salud Mental Positiva, los estudios existentes conducen a plantear la hipótesis general de que la población de forma mayoritaria tiene un nivel medio-alto de Salud Mental Positiva y que existen diferencias según determinantes sociodemográficos, laborales y de salud en los niveles de salud mental positiva global y por factores.

\section{Estrategias para cuidar la Salud Mental Positiva}

A partir de los factores del modelo MMSMP, se planteó un Decálogo de Salud Mental Positiva. Un conjunto de 10 recomendaciones que sirven para operativizar el modelo multifactorial, aportando sugerencias para cuidar la salud mental positiva propia y de los demás (Lluch-Canut, 2011). Está disponible en tres idiomas en http://diposit.ub.edu/dspace/handle/2445/20062.

Recientemente, en base a este decálogo se han elaborado y publicado diversos Decálogos de Salud Mental Positiva adaptados a situaciones y/ o personas afectadas por la pandemia del Coronavirus COVID-19 (Lluch-Canut, 2020). En concreto, se han elaborado Decálogos para Profesionales de la Salud en situaciones de Emergencia (Mantas-Jiménez, 2020), para personas en situación de confinamiento (Puig-Llobet, 2020), para adolescentes (Brando-Garrido, 2020), para gestantes Biurrun-Garrido, 2020) y para gente mayor (Brando-Garrido, 2020).

Una línea estratégica muy interesante son los estudios que correlacionan la Salud Mental Positiva con la capacidad de Autocuidado de las personas postulando un modelo bidireccional de ambos constructos (Albacar-Riobóo, 2014; Miguel, 2014; Sánchez, 2015). Los estudios mostraron una correlación positiva de forma que "a más autocuidado mejor salud mental positiva y a mejor salud mental positiva más capacidad de autocuidado". Esta relación bidireccional es muy importante porque en ocasiones las estrategias para potenciar la salud mental positiva pueden iniciarse a partir de cuidados de salud básicos (comer, dormir, pasear, hidratar la piel,..) que generan sentimientos de bienestar y estimulan a la persona incrementando la satisfacción personal, las ganas de establecer y 
potenciar relaciones sociales, la energía para resolver problemas, entre otros. Y por otro lado, por ejemplo, si se generan estrategias para potenciar el bienestar emocional y la ilusión también repercuten en las ganas para cuidarse más (comer mejor, hacer más ejercicio,...). Es la relación bidireccional entre Autocuidado y Salud Mental Positiva.

Por otro lado, están surgiendo diversos Programas de Intervención para Cuidar la Salud Mental Positiva que tienen por objeto generar estrategias de intervención que puedan ser de utilidad a personas en situaciones específicas de vida. Hay varios programas en construcción y en fase de validación. Los que ya han sido publicados en que he participado son: Programa de Intervenciones Psicosociales Enfermeras para Potenciar la Salud Mental Positiva y el Autocuidado (PIPsE) en personas con problemas crónicos de salud física (Sánchez, 2015; Sánchez-Ortega et al., 2015), Programa para Enseñar Salud Mental Positiva a Estudiantes de Grado de Enfermería (Ferré-Grau et al., 2014), Programa TIVA de Salud Mental Positiva para cuidadoras de personas con problemas crónicos de salud a través de una APP (Ferré-Grau, 2019), y el Programa de Salud Mental Positiva aplicado al ámbito laboral en múltiples tipos y contextos de trabajo (Teixeira et al., 2019, 2020).

\section{Reflexiones finales a modo de conclusiones}

Este apartado debe ir por fuerza dedicado de forma específica a la situación actual en la que se encuentra inmersa el ser humano: la pandemia COVID-19 y el paso a "una nueva normalidad" que, desde mi punto de vista, y tras lo expuesto, precisa reflexionar en torno a dos aspectos de la Salud Mental Positiva: el desarrollo existencial y el Sufrimiento Emocional

La Salud Mental Positiva en el contexto de desarrollo existencial del ser humano.

Reflexionando, y recurriendo a la historia de la humanidad, no podemos pensar que ésta sea la peor situación que el ser humano ha vivido a lo largo de su existencia, pero está claro que cada generación vivimos la vida según las circunstancias concretas. La historia nos puede ayudar y mucho a comprender el momento actual, aunque ninguna vivencia pasada pueda servir para substituir la presente. Cada persona vive la experiencia de vivir como única y propia. Y, tal y como se destacaba al inicio de este artículo, será de gran relevancia el modo en que cada persona pueda vivir la vida en esta realidad actual. Desde la Salud Mental Positiva se plantea un enfoque donde la persona de espacio a toda la complejidad mental de sentimientos, emociones, pensamientos positivos y negativos pero que sea capaz de experimentar un cierto grado de: a) satisfacción personal consigo misma y con su vida; b) sensibilidad social hacia su entorno y hacía la diversidad de modos de vida y de respuestas humanas; c) autocontrol de las emociones, especialmente de las emociones negativas o que generan malestar; d) autonomía para tomar sus propias decisiones; e) capacidad para resolver problemas y para mirar hacia el futuro con deseos de auto-actualizarse y no quedarse anclado en el pasado; y f) capacidad para establecer y 
mantener relaciones sociales armoniosas, comunicarse de forma afectiva y crear relaciones íntimas y amorosas (Lluch-Canut, 1999, 2002, 2008, 2015).

Recogiendo los contenidos que se han descrito a lo largo del artículo se puede concluir con una idea que refleja de forma fácil muchos de los aspectos que se han señalado. Se podría decir que "la Salud Mental Positiva es un coctel de emociones, pensamientos, sentimientos y conductas, positivas y negativas. Todos los ingredientes son necesarios. Pero para que el coctel este en su punto hay que ajustar las dosis" (LluchCanut, 2020 en Facebook). Está definición, en cierto grado teñida de humor, ha de entenderse con carácter totalmente respetuoso y en un contexto distendido.

Mención aparte son las situaciones en que las personas están en sufrimiento emocional y por ello, no podría terminar el trabajo de Salud Mental Positiva sin aportar algunos aspectos de máxima relevancia para situaciones de sufrimiento que algunas personas están viviendo en estos momentos de pandemia COVID-19.

\section{La Salud Mental Positiva y el Sufrimiento Emocional del ser humano}

El sufrimiento emocional es un constructo con múltiples definiciones, pero todas las personas que sufren a nivel emocional saben el significado del término, aunque no pueda expresarse con palabras o aunque las palabras para expresarlo sean diferentes de unas personas a otras.

En esta época de pandemia COVID-19 una de las causas principales de sufrimiento emocional está vinculada a la pérdida de personas queridas. Perder seres queridos es altamente doloroso a nivel emocional. El corazón se rompe simbólicamente y la salud mental positiva tiembla. Pero no hay que tener miedo a llorar y a sentir. Hay que darse permiso absoluto para experimentar cualquier sentimiento y cualquier emoción. $Y$ también hay que darse permiso para funcionar en el día a día al ritmo que se pueda. Pero desde la Salud Mental Positiva señalamos la importancia de estar atento al máximo a los indicadores de alerta de la Salud Mental Positiva que se han descrito en anteriores apartados. Si la tristeza y el sufrimiento son tan intensos que nos bloquean las funciones básicas de comer (aunque sea poco y suave), dormir (aunque sean menos horas de lo habitual), comunicarnos mínimamente con nuestro entorno más inmediato e ir progresivamente avanzando en la continuidad de los requerimientos de la vida, hay que vigilar y pedir ayuda si hace falta. Pero no hay que tener miedo al sufrimiento ya que es normal y sano: no se pueden despedir personas queridas sin sufrir el dolor emocional de la pérdida. El sufrimiento emocional en muchas ocasiones es una gran señal de una buena Salud Mental Positiva.

Otra de las causas principales de sufrimiento emocional en esta época de pandemia COVID-19 es el miedo (a lo que puede suceder en el futuro a nivel social, laboral o económico, a lo que puede suceder a cada persona y a su entorno más inmediato a nivel de salud y de bienestar, etc.). También el miedo es una respuesta emocional que genera malestar pero que es muy sana mentalmente. $Y$, al igual que en el sufrimiento emocional por pérdida de personas queridas, en el miedo también hay que controlar los indicadores 
de alerta de la Salud Mental Positiva vigilando que la intensidad del miedo no nos bloquee y no impida seguir funcionando y tirando hacía adelante en la vida. Y procurando que el miedo no invada al completo nuestra vida, buscando espacios y momentos para vivir el aquí y el ahora buscando la percepción de algunas pequeñas cosas buenas que cada situación puede tener. Tal y como he señalado al inicio de este artículo, el interés por el estudio de la Salud Mental Positiva surgió a partir del trabajo en el contexto clínico y es en situaciones muy críticas de salud, viendo que incluso en esos momentos, hay personas que son capaces de encontrar la parte de Salud Mental Positiva que haya en la situación específica que se esté viviendo, incluso en fases terminales, si hay conciencia plena. Quienes hayan pasado por estas situaciones pueden entenderlo rápidamente, quienes lo hemos observado lo podemos corroborar y quienes aún no lo han experimentado les invitamos a practicar la búsqueda activa de la Salud Mental Positiva, aplicando en cada momento de la vida un eslogan elaborado a partir del sistema de creencias y valores personales, a partir de la idea de que el ser humano a lo largo de su vida experimentará sentimientos, pensamientos y emociones positivas y negativas, y vivirá situaciones agradables y situaciones difíciles y/o dolorosa. Gestionarlos lo mejor posible para beneficio propio y ajeno es el arte de vivir con el máximo nivel posible de Salud Mental Positiva.

\section{Referencias bibliográficas}

ALBACAR-RIOBOO, N. (2014). Atenció d'infermeria a la cuidadora principal de persones amb esquizofrenia. Valoració dels requisits d'autocura i de salut mental positiva.

Tesis doctoral. Universidad Rovira i Virgili, Tarragona. Disponible en: https://tdx.cat/handle/10803/294732\#page=1

BIURRUN-GARRIDO, A. (2020). "Decálogo de Salud Mental Positiva para Gestantes". En Lluch-Canut, M.T. (Coord.). Decálogos de Salud Mental Positiva adaptados a diferentes situaciones y/o persones afectadas por circunstancias de la pandemia de Coronavirus COVID-19 (pp. 14-16). Barcelona: Colección OMADO, Deposito Digital de la Universidad de Barcelona. Disponible en: http://hdl.handle.net/2445/155018

BRANDO-GARRIDO, C. (2020). "Decálogo de Salud Mental Positiva para Adolescente". En Lluch-Canut, M.T. (Coord.). Decálogos de Salud Mental Positiva adaptados a diferentes situaciones y/o personas afectadas por circunstancias de la pandemia de Coronavirus COVID-19 (pp. 11-13). Barcelona: Colección OMADO, Deposito Digital de la Universidad de Barcelona. Disponible en: http://hdl.handle.net/2445/155018

BRANDO-GARRIDO, C. (2020). "Decálogo de Salud Mental Positiva para Personas Mayores". En Lluch-Canut, M.T. (Coord.). Decálogos de Salud Mental Positiva adaptados a diferentes situaciones y/o personas afectadas por circunstancias de la pandemia de Coronavirus COVID-19 (pp. 17-19). Barcelona: Colección OMADO, 
Deposito Digital de la Universidad de Barcelona. Disponible en: http://hdl.handle.net/2445/155018

FERRÉ-GRAU, C.; SOARES DE CARVALHO, D.; PALOMINO-MARTINEZ, A.; BERBIS-MORELLÓ, C.; LLUCH-CANUT, M. T. (2014). Programa de enseñanza de la Salud Mental Positiva a estudiantes de Grado de Enfermería. Tarragona: Repositorio Institucional de la Universidad Rovira i Virgili (Tarragona). Disponible en: http://hdl.handle.net/20.500.11797/DD13

FERRÉ-Grau, C.; RAIGAL-ARAN, L.; LORCA-CABRERA, J.; FERRÉ-BERGADÁ, M.; LLEIXÁFORTUÑO, M. d. M.; LLUCH-CANUT, M. T.; PUIG-LLOBET, M.; ALBACAR-RIOBOO, N. (2019). "A multi-centre, randomized, 3-month study to evaluate the efficacy of a smartphone app to increase caregiver's positive mental Health". En BMC Public Health, 19:888. Disponible en: https://bmcpublichealth.biomedcentral.com/articles/10.1186/s12889-019-7264-5 HURTADO, B. (2017). Niveles de exposición a factores de riesgo psicosocial y la salud mental positiva en docentes universitarios de Enfermería de Cataluña. Tesis doctoral Universidad de Barcelona. Disponible en: https://tdx.cat/handle/10803/403710\#page=1

HURTADO, B.; MORENO, C.; CASAS, I.; LLUCH, M. T.; LLEIXÀ, M. d. M.; FARRËS, M.; ROLDAN, J.. (2017). "Positive Mental Health and Prevalence of Psychological III-Being in University Nursing Professors in Catalonia, Spain". En Journal of Psychosocial Nursing and Mental Health Services, 1;55(7):38-48.

HURTADO, B.; LLUCH, M. T.; CASAS, I.; SEQUEIRA, C.; PUIG, M.; ROLDAN, J. F. (2018). "Evaluación de la fiabilidad y validez del cuestionario de salud mental positiva en profesores universitarios de enfermería en Cataluña". En Revista de Enfermería y Salud Mental, 9, 5-17.

JAHODA, M. (1958). Current concepts of positive mental health. Nueva York: Basic Book.

LLUCH-CANUT, M. T. (1999). "Construcción de una escala para evaluar la salud mental positiva". Tesis doctoral. Universidad de Barcelona. Disponible en: https://www.tdx.cat/handle/10803/2366;jsessionid=4F9BB85EFA03E62406E9E299B 241BC5F\#page $=1$

LLUCH-CANUT, M. T. (2002). "Promoción de la salud mental: cuidarse para cuidar mejor". En Matronas: profesión, 10-14. Disponible en: http://hdl.handle.net/2445/29057

LLUCH-CANUT, M. T. (2003). "Construcción y análisis psicométrico de un cuestionario para evaluar la salud mental positiva". En Psicología Conductual, 11(1):61-78.

LLUCH-CANUT, M. T. (2008). “Concepto de salud mental positiva: factores relacionados. En J. Fornes y J. Gómez (coord..), Recursos y programas para la salud mental. Enfermería psicosocial II (pp. 37-69). Madrid: Fuden, Colección líneas de especialización en enfermería.

LLUCH-CANUT, M. T. (2011). Decalogue of Positive Mental Health/Decálogo de Salud Mental Positiva. Barcelona: Deposito Digital de la Universidad de Barcelona. 
Disponible

http://diposit.ub.edu/dspace/bitstream/2445/20062/11/Decalogo_Salud_Mental_P ositiva_trilingue.pdf

LLUCH-CANUT, M. T. (2015). "Promoción de la salud mental positiva". En Revista de Enfermería y Salud Mental, 1: 16-22. Disponible en: https://dialnet.unirioja.es/servlet/articulo?codigo $=6145927$

LLUCH-CANUT, M. T. (2020) (Coord.). Decálogos de Salud Mental Positiva adaptados a diferentes situaciones y/o personas afectadas por circunstancias de la pandemia de Coronavirus COVID-19. Barcelona: Colección OMADO, Deposito Digital de la Universidad de Barcelona. Disponible en: http://hdl.handle.net/2445/155018

LLUCH-CANUT, M. T. (2020). WEBinar Resiliencia y Salud Mental. Disponible en Facebook: https://m.facebook.com/story.php?story_fbid=10216247764214413\&id=146208598 7

LLUCH-CANUT, M. T.; PUIG-LLOBET, M.; SÁNCHEZ-ORTGEA, M. A.; ROLDÁN-MERINO, J. F.; FERRÉ-GRAU, C.; POSITIVE MENTAL HEALTH RESEARCH GROUP (PMHRG) (2013). "Assessing positive mental health in people with chronic physical health problems: correlations with socio-demographic variables and physical health status". En BMC Public Health, 13:928. Disponible en: https://pubmed.ncbi.nlm.nih.gov/24093443/

LLUCH-CANUT, M. T.; SEQUEIRA, C.; ROLDÁN-MERINO, J. F. (2017). "La salud mental positiva desde una perspectiva multifactorial". En V. Fragoso y M. Sotto Mayor (Coords.), Gerontologia e transdisciplinariedade I (pp. 114-129). Sâo Paulo (Brasil): Portal Ediçôes Envelhecimento.

MANTAS JIMENEZ, S. (2017). Sentido de coherencia, resiliencia y salud mental positiva en profesionales del Sistema de Emergencias Médicas. Tesis doctoral. Universidad de Gerona. Disponible en: https://tdx.cat/handle/10803/406045\#page=1

MANTAS JIMENEZ, S. (2020). “Decálogo de Salud Mental Positiva para los Profesionales de la Salud en situaciones de Emergencia". En Lluch-Canut, M.T. (Coord.). Decálogos de Salud Mental Positiva adaptados a diferentes situaciones y/o personas afectadas por circunstancias de la pandemia de Coronavirus COVID-19 (pp. 5-7). Barcelona: Colección OMADO, Depósito Digital de la Universidad de Barcelona. Disponible en: http://hdl.handle.net/2445/155018

MANTAS JIMENEZ, S.; JUVINYÀ i CANA, D.; BERTRAN I NOGUER, C.; ROLDÁN MERINO, J. F.; SEQUEIRA, C.; LLUCH-CANUT, M. T. (2015). "Evaluation of positive mental health and sense of coherence in mental health professionals". En Revista Portuguesa de Enfermagem de Saúde Mental, (13):34-42. Disponible en: http://www.scielo.mec.pt/scielo.php?script=sci_arttext\&pid=S1647$21602015000200005 \&$ lng =pt\&nrm=iso\&tlng=en

MIGUEL, M. D. (2014). Valoración de la Salud Mental Positiva y de los Requisitos de Autocuidado, en pacientes hospitalizados diagnosticados de Esquizofrenia, según la Teoría de Enfermería de Dorothea Orem. Tesis Doctoral. Universidad de Barcelona. Disponible en: https://tdx.cat/handle/10803/275959\#page=1 
PUIG LLOBET, M. (2020). "Decálogo de Salud Mental Positiva para personas en situación de confinamiento". En Lluch-Canut, M.T. (Coord.). Decálogos de Salud Mental Positiva adaptados a diferentes situaciones $\mathrm{y} / \mathrm{o}$ personas afectadas por circunstancias de la pandemia de Coronavirus COVID-19 (pp. 8-10). Barcelona: Colección OMADO, Depósito Digital de la Universidad de Barcelona. Disponible en: http://hdl.handle.net/2445/155018

ROLDAN, J.; LLUCH, M. T.; CASAS, I.; SANROMÀ, M.; FERRÉ, C.; SEQUEIRA, C.; FALCO, A.; SOARES, D.; PUIG, M. (2017). "Reliability and validity of the Positive Mental Health Questionnaire in a sample of Spanish university students". En Journal of Psychosocial and Mental Health Nursing, 24 (2-3):123-133.

SÁNCHEZ, M. A. (2015). Efectividad de un programa de intervención psicosocial enfermera para potenciar la agencia de autocuidado y la salud mental positiva en personas con problemas crónicos de salud. Tesis doctoral. Universidad de Barcelona. Disponible en:

https://www.tdx.cat/bitstream/handle/10803/387116/MASO_TESIS.pdf?sequence= 1\&isAllowed $=y$

SÁNCHEZ-ORTEGA, M. A.; PUIG-LlOBET, M.; LLUCH-CANUT, M. T. (2015). Programa d'Intervenció Psicosocial d'Infermeria (PIPsI) per a potenciar la Salut Mental Positiva i l'Autocura. Barcelona: Depósito Digital de la Universidad de Barcelona, Colección OMADO. Disponible en: https://tdx.cat/handle/10803/387116\#page=1

SANROMÁ ORTIZ, M. (2016). Evaluación de un programa de empoderamiento para potenciar y desarrollar conductas promotoras de salud y salud mental positiva en estudiantes de enfermería. "Dinamiza la Salud: Cuídate y Cuida". Tesis doctoral Universidad de Barcelona. Disponible en: https://tdx.cat/handle/10803/386481\#page=1

SEQUIERA, C.; CARVALHO, J. C.; SAMPAIO, F.; SÁ, L.; LLUCH-CANUT, M. T.; ROLDÁNMERINO, J. F. (2014). "Avaliação das propriedades psicométricas do Questionário de Saúde Mental Positiva em estudantes portugueses do ensino superior". En Revista Portuguesa de Enfermagem de Saúde Mental, (11), 45-53. http://www.scielo.mec.pt/pdf/rpesm/n11/n11a07.pdf

SEQUEIRA, C.; CARVALHO, J. C.; GONÇALVOS, A.; NOGUEIRA, M. J.; LLUCH-CANUT, M. T. (2019 online). "Levels of Positive Mental Health in Portuguese and Spanish Nursing Students". En Journal of American Psychological Nurses Association.

TEIXEIRA ALMEIDA, S.; COELHO FERREIRA, J.; SEQUEIRA DA CRUZ, C.; LLUCH-CANUT, M. Te.; FERRÉ-GRAU, C. (2019). "The effectiveness of positive mental health programs in adults: A systematic review". En Health and Social Care, 27; 1126-1134.

TEIXEIRA, S.; SEQUEIRA, C.; LLUCH-CANUT, M. T.; FERRÉ-GRAU, C. (2020). Programa de Salud Mental Positiva para Adultos. Tesis doctoral en curso. Tarragona: Universitat Rovira i Virgili. 
TEKE, C.; BAYSAN ARABACI, L. (2018). "The validity and reliability of Positive Mental Health Scale". En Anadolu Psikiyatri Derg, 19,21-28.

* María Teresa Lluch Canut: Diplomada en Enfermería, especialista en Enfermería de Salud Mental. Licenciada en Filosofía y Letras- Sección Psicología y Doctora en Psicología por la Universidad de Barcelona (España). Autora del Modelo Multifactorial de Salud Mental Positiva, del Cuestionario de Salud Mental Positiva y del Decálogo de Salud Mental Positiva. Profesora Catedrática de Enfermería Psicosocial y Salud Mental en la Universidad de Barcelona (España). [E-mail: tlluch@ub.edu] 